TMUP-HEL-0003

hep-ph/0004114

April 2000, Revised August 2000

\title{
Measuring Leptonic CP Violation by Low Energy Neutrino Oscillation Experiments
}

\author{
Hisakazu Minakata * \\ Department of Physics, Tokyo Metropolitan University \\ 1-1 Minami-Osawa, Hachioji, Tokyo 192-0397, Japan, and \\ Research Center for Cosmic Neutrinos, Institute for Cosmic Ray Research, \\ University of Tokyo, Kashiwa, Chiba 277-8582, Japan \\ Hiroshi Nunokawa ${ }^{\dagger}$ \\ Instituto de Física Gleb Wataghin \\ Universidade Estadual de Campinas - UNICAMP \\ P.O. Box 6165, 13083-970 Campinas SP Brazil
}

\begin{abstract}
We uncover an interesting phenomenon that neutrino flavor transformation in slowly varying matter density imitates almost exactly that of vacuum neutrino oscillation under suitably chosen experimental parameters. It allows us to have relatively large $\mathrm{CP}$ violating measure $\Delta P \equiv P\left(\nu_{\mu} \rightarrow \nu_{e}\right)-P\left(\bar{\nu}_{\mu} \rightarrow\right.$ $\left.\bar{\nu}_{e}\right)$ which is essentially free from matter effect contamination. We utilize this phenomenon to design a low-energy long-baseline neutrino oscillation experiment to measure the leptonic $\mathrm{CP}$ violating phase.
\end{abstract}

\footnotetext{
*E-mail: minakata@phys.metro-u.ac.jp

${ }^{\dagger}$ E-mail: nunokawa@ifi.unicamp.br
} 
Exciting discovery of neutrino oscillation in atmospheric neutrino observation [1] and the persistent discrepancy between the observed and the calculated flux of solar neutrinos [2] provide the strongest evidence for neutrino masses and lepton flavor mixing. Determination of all the mixing parameters, in particular the CP violating Kobayashi-Maskawa phase [3], is one of the most challenging goals in particle physics.

This is the third in a series of works [4,5] in which we intend to explore possible (and hopefully experimentally feasible) ways of measuring leptonic CP violation in neutrino oscillation experiments. For early references and recent works on $\mathrm{CP}$ violation (or equivalently T violation), see for example, Refs. [6,7] and Refs. [8.9], respectively. We focus in this paper the neutrino mass (difference) hierarchy suggested by the atmospheric neutrino observation and the MSW solutions [10] of the solar neutrino problem [8]. In the standard three-flavor mixing scheme of neutrinos they exhaust all the independent mass difference squared: from atmospheric neutrino data, $\Delta m_{13}^{2} \approx \Delta m_{23}^{2}=\Delta m_{\text {atm }}^{2} \simeq(2-5) \times 10^{-3} \mathrm{eV}^{2}$ and from solar neutrino data, $\Delta m_{12}^{2}=\Delta m_{\text {solar }}^{2} \simeq(4-10) \times 10^{-6} \mathrm{eV}^{2}(\mathrm{SMA}),(2-20) \times 10^{-5} \mathrm{eV}^{2}(\mathrm{LMA})$ or $(6-20) \times 10^{-8} \mathrm{eV}^{2}(\mathrm{LOW})$ where SMA, LMA, and LOW denote the small mixing angle, large mixing angle and the low $\Delta m^{2}$ MSW solutions, respectively [11,12]. We use the notation in this paper as $\Delta m_{i j}^{2} \equiv m_{j}^{2}-m_{i}^{2}$.

Many authors including us examined the question of how to separate the genuine $\mathrm{CP}$ violating effect due to the leptonic Kobayashi-Maskawa phase from the fake one induced by matter effect [4,5, 8, 9]. In this paper we take a simple alternative strategy to look for a region of parameters in which the matter effect is "ignorable" in the first approximation. More precisely speaking, we will look for the solution of the question; is there region of tunable parameters in experiments, such as energy of neutrino beam, baseline length, etc. in which the neutrino oscillation probability, including its CP-odd term, are dominated by vacuum mixing effects? (See below on what we mean precisely by "dominated by vacuum mixing effects".) We will answer the question in the positive and finally end up with a proposal of experiment which utilizes low-energy neutrino beam of $E \sim 100 \mathrm{MeV}$ and a megaton water Cherenkov detector to measure leptonic $\mathrm{CP}$ violation.

We define the flavor mixing matrix $U$ as $\nu_{\alpha}=U_{\alpha i} \nu_{i}$, where $\nu_{\alpha}(\alpha=e, \mu, \tau)$ and $\nu_{i}(i=$ $1,2,3)$ stand for the gauge and the mass eigenstates, respectively. We take for convenience the representation of $U$ as

$$
\begin{gathered}
U=e^{i \lambda_{7} \theta_{23}} \Gamma_{\delta} e^{i \lambda_{5} \theta_{13}} e^{i \lambda_{2} \theta_{12}} \\
=\left[\begin{array}{ccc}
c_{12} c_{13} & s_{12} c_{13} & s_{13} \\
-s_{12} c_{23}-c_{12} s_{23} s_{13} e^{i \delta} & c_{12} c_{23}-s_{12} s_{23} s_{13} e^{i \delta} & s_{23} c_{13} e^{i \delta} \\
s_{12} s_{23}-c_{12} c_{23} s_{13} e^{i \delta} & -c_{12} s_{23}-s_{12} c_{23} s_{13} e^{i \delta} & c_{23} c_{13} e^{i \delta}
\end{array}\right],
\end{gathered}
$$

where $\lambda_{i}$ are $\mathrm{SU}(3)$ Gell-Mann's matrix and $\Gamma_{\delta}=\operatorname{diag}\left(1,1, e^{i \delta}\right)$.

Let us first assume that the matter effect plays a minor role and consider the $\mathrm{CP}$ violating effect in vacuum. We will justify this assumption later. Under the mass difference hierarchy $\Delta m_{13}^{2} \gg \Delta m_{12}^{2}$ which is implied by the solar and atmospheric neutrino data, the neutrino oscillation probability in vacuum can be written as

$$
\begin{aligned}
P\left(\nu_{\beta} \rightarrow \nu_{\alpha}\right)= & 4\left|U_{\alpha 3}\right|^{2}\left|U_{\beta 3}\right|^{2} \sin ^{2}\left(\frac{\Delta_{13} L}{2}\right)-4 \operatorname{Re}\left[U_{\alpha 1} U_{\alpha 2}^{*} U_{\beta 1}^{*} U_{\beta 2}\right] \sin ^{2}\left(\frac{\Delta_{12} L}{2}\right) \\
& -2 J \sin \left(\Delta_{12} L\right)\left[1-\cos \left(\Delta_{13} L\right)\right]+4 J \sin \left(\Delta_{13} L\right) \sin ^{2}\left(\Delta_{12} L\right),
\end{aligned}
$$


where $\Delta_{i j} \equiv \frac{\Delta m_{i j}^{2}}{2 E}$. One of the most significant feature of (3) is that $\mathrm{CP}$ violation comes in through $J$ defined by

$$
J_{\alpha \beta ; i, j} \equiv \operatorname{Im}\left[U_{\alpha i} U_{\alpha j}^{*} U_{\beta i}^{*} U_{\beta j}\right]
$$

as it is the unique (in three-flavor mixing scheme) measure for CP violation as first observed by Jarlskog [13 in the case of quark mixing. It takes the form in the parametrization we introduced above as $J= \pm c_{12} s_{12} c_{23} s_{23} c_{13}^{2} s_{13} \sin \delta$, where the sign is positive for $(e, \mu)$ and $(1,2)$ and $+(-)$ corresponds to their (anti-) cyclic permutations of $(\alpha, \beta)$ and $(i, j)$.

We first observe that from the expression of $J$, if any one of the mixing angles is extremely small or very close to $\pi / 2$ there is little hope in detecting the leptonic CP violation. For this reason, we will not deal with the case of SMA MSW solar neutrino solution. See, however, a remark at the end of this paper.

We also notice that in order to have appreciable effect of $\mathrm{CP}$ violation,

$$
\Delta_{12} L=0.26\left(\frac{\Delta m_{12}^{2}}{10^{-5} \mathrm{eV}^{2}}\right)\left(\frac{L}{1000 \mathrm{~km}}\right)\left(\frac{E}{100 \mathrm{MeV}}\right)^{-1},
$$

should not be too small. If $\Delta m_{12}^{2} \equiv \Delta m_{\text {solar }}^{2}$ is much smaller than $10^{-5} \mathrm{eV}^{2}$ we have to either use a neutrino energy much lower than $\sim 100 \mathrm{MeV}$ which would be impractical in accelerator experiments, or take baseline length much longer than 1,000 km at the cost of lowering the beam flux. For this reason, our discussion can accommodate neither the just-so vacuum oscillation solution nor the LOW MSW solution. Thus we assume in the following discussion that the nature chooses the LMA MSW solution to the solar neutrino problem.

If we restrict ourselves into relatively short baseline, i.e., less than 1,000 km, one can safely assume that linear approximation $\sin \left(\Delta_{12} L\right) \simeq \Delta_{12} L$ is valid. Therefore, in order that the $\mathrm{CP}$ violating (third) term in (3) is relatively larger than the 2nd term we must focus on the region where $\Delta_{12} L$ is smaller but not too smaller than unity. This consideration naturally leads us to the option of low-energy neutrino oscillation experiments with energy of order $\sim 100 \mathrm{MeV}$.

Next we must justify our assumption that the matter effect plays a minor role even for the $\mathrm{CP}$ violating effect. What we will show below is actually that whereas the Jarlskog factor and the energy eigenvalues are strongly modified by the matter effect it almost cancels out and does not show up in the observable quantities, the oscillation probabilities.

We now discuss full system of three-flavor neutrino propagation in earth matter to understand the phenomenon of matter neutrino oscillation imitating vacuum oscillation. Toward this goal we develop an analytic framework based on perturbation theory under the adiabatic approximation [14, 15]. We start from the neutrino evolution equation in the flavor basis:

$$
i \frac{d}{d x}\left[\begin{array}{l}
\nu_{e} \\
\nu_{\mu} \\
\nu_{\tau}
\end{array}\right]=\left\{U\left[\begin{array}{ccc}
0 & 0 & 0 \\
0 & \Delta_{12} & 0 \\
0 & 0 & \Delta_{13}
\end{array}\right] U^{\dagger}+\left[\begin{array}{ccc}
a(x) & 0 & 0 \\
0 & 0 & 0 \\
0 & 0 & 0
\end{array}\right]\right\} \times\left[\begin{array}{c}
\nu_{e} \\
\nu_{\mu} \\
\nu_{\tau}
\end{array}\right]
$$

where $a(x)=\sqrt{2} G_{F} N_{e}(x)$ indicates the index of refraction with $G_{F}$ and $N_{e}$ being the Fermi constant and the electron number density, respectively. 
We first note that at neutrino energy of $\sim 100 \mathrm{MeV}$ there is a hierarchy among the relevant energy scales;

$$
\Delta_{13} \equiv \frac{\Delta m_{13}^{2}}{2 E} \gg a \sim \frac{\Delta m_{12}^{2}}{2 E} \equiv \Delta_{12}
$$

The latter relationship holds because

$$
\frac{a(x)}{\Delta m^{2} / 2 E}=2.1\left(\frac{\rho}{2.7 \mathrm{~g} / \mathrm{cm}^{3}}\right)\left(\frac{Y_{e}}{0.5}\right)\left(\frac{\Delta m^{2}}{10^{-5} \mathrm{eV}^{2}}\right)^{-1}\left(\frac{E}{100 \mathrm{MeV}}\right) .
$$

Thanks to the mass hierarchy (7) we can formulate the perturbation theory.

We rewrite the evolution equation (6) as [16]

$$
i \frac{\mathrm{d}}{\mathrm{d} x} \tilde{\nu_{\alpha}}=\left(H_{0}+H^{\prime}\right)_{\alpha \beta}, \tilde{\nu_{\beta}}
$$

where $\tilde{\nu}$ is defined by

$$
\tilde{\nu_{\alpha}}=\left[e^{-i \lambda_{5} \theta_{13}} \Gamma_{\delta}^{+} e^{-i \lambda_{7} \theta_{23}}\right]_{\alpha \beta} \nu_{\beta},
$$

and $H_{0}=\operatorname{diag}\left(0,0, \Delta_{13}\right)$ and

$$
H^{\prime}=\Delta_{12}\left[\begin{array}{ccc}
s_{12}^{2} & c_{12} s_{12} & 0 \\
c_{12} s_{12} & c_{12}^{2} & 0 \\
0 & 0 & 0
\end{array}\right]+a(x)\left[\begin{array}{ccc}
c_{13}^{2} & 0 & c_{13} s_{13} \\
0 & 0 & 0 \\
c_{13} s_{13} & 0 & s_{13}^{2}
\end{array}\right] .
$$

The diagonalization of the $2 \times 2$ submatrix gives rise to the energy eigenvalues $h_{i}$ and the matter enhanced $\theta_{12}$ as

$$
\begin{gathered}
h_{1,2}=\frac{1}{2}\left[c_{13}^{2} a(x)+\Delta_{12} \mp \sqrt{\left(\cos 2 \theta_{12} \Delta_{12}-a c_{13}^{2}\right)^{2}+\Delta_{12}^{2} \sin ^{2} 2 \theta_{12}}\right], \\
\sin 2 \theta_{12}^{M}=\frac{\sin 2 \theta_{12}}{\sqrt{\left(\cos 2 \theta_{12}-\frac{a}{\Delta_{12}} c_{13}^{2}\right)^{2}+\sin ^{2} 2 \theta_{12}}}
\end{gathered}
$$

The matter "suppressed" $\bar{\theta}_{12}^{M}$ for antineutrinos and the energy eigenvalues can be defined analogously by flipping the sign of $a$ in (12) and (13). The resonance condition is satisfied in neutrino channel tat

$$
c_{13}^{2} a(x)=\cos 2 \theta_{12} \Delta_{12}
$$

which leads to the resonance energy of order of $100 \mathrm{MeV}$,

\footnotetext{
${ }^{1}$ We note that one can take $\Delta m_{12}^{2}$ always positive in the MSW mechanism as far as $\theta_{12}$ is taken in its full range $[0, \pi / 2]$ [12].
} 


$$
E_{r}=48.6 \cos 2 \theta_{12}\left(\frac{\Delta m_{12}^{2}}{10^{-5} \mathrm{eV}^{2}}\right)\left(\frac{\rho}{2.7 \mathrm{~g} / \mathrm{cm}^{3}}\right)^{-1}\left(\frac{Y_{e}}{0.5}\right) \mathrm{MeV}
$$

for small value of the remaining vacuum mixing angle, $\theta_{13} \ll 1$. For the best fit values of the parameter of the LMA MSW solution, $\Delta m_{12}^{2} \sim(3.0-4.0) \times 10^{-5} \mathrm{eV}^{2}$ and $\sin ^{2} 2 \theta_{12} \sim 0.8$ [11], we obtain $E_{r} \sim 60-80 \mathrm{MeV}$.

The key to the matter enhanced $\theta_{12}$ mechanism is the degeneracy of the zeroth order "energy" eigenvalue in the first $2 \times 2$ subspace of $H_{0}$. The degenerate perturbation theory dictates that one has to first diagonalize $H_{0}+H^{\prime}$ in this subspace to obtain the first-order corrected energy eigenvalues and the zeroth-order wave function. This last point is crucial. It gives rise to the matter enhanced $\mathrm{CP}$ violating effect that is free from the suppression by energy denominator. To our knowledge, this is the unique case of having $\mathrm{CP}$ violating effect which is not suppressed by any hierarchical ratios such as $\Delta m_{12}^{2} / \Delta m_{13}^{2}$

While our formalism is exactly the same as the one developed in Ref. [14 the interpretation of the physical phenomena that occur is quite different from theirs. Also the nature of the resonance is quite different in large mixing angles. The resonance width can be estimated as $\frac{\delta E}{E_{r}}=\tan 2 \theta_{12}$ which means that $\delta E \simeq 2 E_{r} \simeq 120 \mathrm{MeV}$. Therefore, the resonances are so broad that they lose the identity as sharp resonances.

We now show that despite the fact that $\theta_{12}$ could be strongly modified in matter, especially for small vacuum $\theta_{12}$, the system mimics the vacuum neutrino oscillation even at the resonance where the effect of matter could be maximal. To understand this point we calculate the neutrino and antineutrino conversion probabilities $P\left(\nu_{\mu} \rightarrow \nu_{e}\right)$ and $P\left(\bar{\nu}_{\mu} \rightarrow \bar{\nu}_{e}\right)$. Since the matter enhanced mixing angle $\theta_{12}^{M}$ just replaces $\theta_{12}$ in zeroth-order wave function it is straightforward to compute neutrino oscillation probabilities under the adiabatic approximation. They are nothing but the oscillation probabilities in vacuum but with $\theta_{12}$ replaced by $\theta_{12}^{M}$, and $\Delta_{i j}$ by integrals over the energy eigenvalues, $h_{1,2}$ and $h_{3} \simeq \Delta_{13}$. For example, the appearance probability $P\left(\nu_{\mu} \rightarrow \nu_{e}\right)$ reads

$$
\begin{aligned}
P\left(\nu_{\mu} \rightarrow \nu_{e}\right)= & 4 s_{23}^{2} c_{13}^{2} s_{13}^{2} \sin ^{2}\left(\frac{1}{2} \Delta_{13} L\right) \\
& +c_{13}^{2} \sin 2 \theta_{12}^{M}\left[\left(c_{23}^{2}-s_{23}^{2} s_{13}^{2}\right) \sin 2 \theta_{12}^{M}+2 c_{23} s_{23} s_{13} \cos \delta \cos 2 \theta_{12}^{M}\right] \\
& \times \sin ^{2}\left[\frac{1}{2} \sqrt{\left.\left(\cos 2 \theta_{12}-\frac{a}{\Delta_{12}} c_{13}^{2}\right)^{2}+\sin ^{2} 2 \theta_{12} \Delta_{12} L\right]}\right. \\
& -2 J_{M}\left(\theta_{12}^{M}, \delta\right) \sin \left[\sqrt{\left(\cos 2 \theta_{12}-\frac{a}{\Delta_{12}} c_{13}^{2}\right)^{2}+\sin ^{2} 2 \theta_{12}} \Delta_{12} L\right]
\end{aligned}
$$

where $J_{M}$ is the matter enhanced Jarlskog factor, $J_{M}\left(\theta_{12}^{M}, \delta\right)=\cos \theta_{12}^{M} \sin \theta_{12}^{M} c_{23} s_{23} c_{13}^{2} s_{13} \sin \delta$, and we have averaged the rapidly oscillating piece driven by $\Delta_{13}$ in the CP violating term. The antineutrino transition probability $P\left(\bar{\nu}_{\mu} \rightarrow \bar{\nu}_{e}\right)$ is given by the same expressions as above but replacing $\theta_{12}^{M}$ by $\bar{\theta}_{12}^{M}$ and $\delta$ by $-\delta$.

Notice that at relatively short baseline, $L<1,000 \mathrm{~km}$ or so, the approximation $\sin x \simeq x$ is valid. Then, the expressions of the oscillation probabilities approximately reduce to those in the vacuum because

$$
\sin 2 \theta_{12}^{M}\left(\text { or, } \bar{\theta}_{12}^{M}\right) \sqrt{\left(\cos 2 \theta_{12} \mp \frac{a}{\Delta_{12}} c_{13}^{2}\right)^{2}+\sin ^{2} 2 \theta_{12}} \Delta_{12}=\sin 2 \theta_{12} \Delta_{12} .
$$


Only mild $a$-dependence would remain due to the $\cos \delta$ term in (16). Hence, as long as $\Delta_{12} L$ is small, the oscillation probabilities of neutrinos and antineutrinos in matter imitate those in vacuum, independent of the mixing parameters and neutrino energy.

We verify by numerical computations without using analytic expression in eq. (16) that the matter effect in fact cancels out. We tentatively take the values of the parameters as $\Delta m_{13}^{2}=3 \times 10^{-3} \mathrm{eV}^{2}, \sin ^{2} 2 \theta_{23}=1.0, \Delta m_{12}^{2}=2.7 \times 10^{-5} \mathrm{eV}^{2}, \sin ^{2} 2 \theta_{12}=0.79, \sin ^{2} 2 \theta_{13}=0.1$ and the Kobayashi-Maskawa phase $\delta=\pi / 2$. Our choice of $\theta_{13}$ is within the newer CHOOZ bound [17], but it may still be an optimistic one. With these parameters the Jarlskog factor in vacuum is given by $J=0.035$, a small but non-negligible value. If we take $\delta=-\pi / 2$ it corresponds, in a good approximation, to interchanging $\nu$ and $\bar{\nu}$ because the matter effect is only minor.

In Fig. 11 we plot the oscillation probabilities for neutrino and anti-neutrino, and their difference, $\Delta P \equiv P\left(\nu_{\mu} \rightarrow \nu_{e}\right)-P\left(\overline{\nu_{\mu}} \rightarrow \overline{\nu_{e}}\right)$, as a function of distance from the source with the neutrino energy $E=60 \mathrm{MeV}$, for both $\nu_{\mu} \rightarrow \nu_{e}$ and $\overline{\nu_{\mu}} \rightarrow \overline{\nu_{e}}$. It corresponds to the resonance energy in the neutrino channel. Insensitivity of the transition probability to the matter effect and how well it mimics the vacuum oscillation probability is clearly displayed in this figure. Although we sit on at the resonance energy of neutrino channel the features in the resonant neutrino flavor conversion cannot be traced in Fig. 1, but rather we observe the one very much similar to the vacuum oscillation. We have also checked that the matter effect is small even if $\cos \delta$ term in (16) is non-zero. The feature that it is a product of two harmonics with quite different frequencies can be understood by (3). We note that a large amplitude of $|\Delta P|$ (as large as $\sim 0.3$ ) in Fig. 11 can be qualitatively understood from the third term in our approximated analytic expression in eq. (3) which implies that $|\Delta P|$ can be as large as $8 J(\sim 0.28)$ at $\Delta_{13} L=\pi$.

Now we discuss the possible experiments which utilize the imitating vacuum mechanism to measure leptonic $\mathrm{CP}$ violation. Measurement of $\mathrm{CP}$ violation at a few $\%$ level at neutrino oscillation experiments at $E \simeq 100 \mathrm{MeV}$ leaves practically the unique channel $\nu_{\mu} \rightarrow \nu_{e}$. Experimentally, what we can actually measure is the number of events not the oscillation probability itself. Here we consider the ratio of the expected number of events due to $\nu_{\mu} \rightarrow \nu_{e}$ and $\overline{\nu_{\mu}} \rightarrow \overline{\nu_{e}}$ reactions in order to quantify the effect of CP violation.

In such low energy appearance experiment we must circumvent the following difficulties; (1) smaller cross sections (2) lower flux due to larger beam opening angle, $\Delta \theta \simeq 1$ (E/100 $\mathrm{MeV})^{-1}$ radian. Therefore, we are invited to the idea of the baseline as short as possible, because the luminosity decreases as $L^{-2}$ as baseline length grows. But of course, a baseline as long as possible is preferable to make the $\mathrm{CP}$ violating effect [the last term in (16)] maximal. Thus we have to compromise. It appears to us that for $E \sim 100 \mathrm{MeV}$, the baseline of 30-50 km seems preferable, as we will see below.

Detection of low-energy neutrinos at a few \% level accuracy requires supermassive detectors. Probably the best thinkable detection apparatus is the water Cherenkov detector of Superkamiokande type. Let us estimate the expected number of events at a megaton detector placed at $L=100 \mathrm{~km}$. We assume that the neutrino beam flux 10 times as intense as (despite the difference in energy) that of the design luminosity in K2K experiment [19]. In the future it is expected that an 100 times more intense proton flux than KEK-ps seems possible at Japan Hadron Facility [20] but we assume only 10 times larger flux considering the fact that lower energy beam is more difficult to prepare. 
The dominant $\nu_{e}$-induced reaction in water at around $E=100 \mathrm{MeV}$ is not the familiar $\nu_{e}-e$ elastic scattering but the reaction on ${ }^{16} O, \nu_{e}^{16} O \rightarrow e^{-} F[18]$. The cross section of the former reaction is about $\sigma\left(\nu_{e} e \rightarrow \nu_{e} e\right)=0.93 \times 10^{-42}\left(\frac{E}{100 \mathrm{MeV}}\right) \mathrm{cm}^{2}$, while the latter is $\sigma\left(\nu_{e}^{16} O \rightarrow e^{-} F\right) \simeq 10^{-39} \mathrm{~cm}^{2}$ at $E=100 \mathrm{MeV}$ [18], which is a factor of 1000 times larger.[ But the number of oxygen in water is $1 / 10$ of the number of electrons. So in net the number of events due to the reaction $\nu_{e}^{16} O \rightarrow e^{-} F$ is larger than that of $\nu_{e} e$ elastic scattering by a factor of 100 . The number of ${ }^{16} \mathrm{O}$ in detector is given by $3.34 \times 10^{31}$ per kton of water. The neutrino flux at the detector located at $L=250 \mathrm{~km}$ is, by our assumption, 10 times more intense than the neutrino flux at Superkamiokande in K2K experiment. The latter is, roughly speaking, $3 \times 10^{6}\left(\frac{\mathrm{POT}}{10^{20}}\right) \mathrm{cm}^{-2}$ where POT stands for proton on target. Therefore, the expected number of events $N$ assuming $100 \%$ conversion of $\nu_{\mu}$ to $\nu_{e}$ is given by

$$
N \simeq 6300\left(\frac{L}{100 \mathrm{~km}}\right)^{-2}\left(\frac{V}{1 \mathrm{Mton}}\right)\left(\frac{\mathrm{POT}}{10^{21}}\right) .
$$

In the antineutrino channel, the dominant reaction is $\bar{\nu}_{e} p \rightarrow e^{+} n$ with cross section $\sigma \simeq 0.4 \times 10^{-39} \mathrm{~cm}^{2}$ at $E=100 \mathrm{MeV}$. The event number due to this reaction, assuming the same flux of $\bar{\nu}_{\mu}$ as $\nu_{\mu}$, is similar to that of (18) because the cross section is about half but there are two free protons per one oxygen. There is additional oxygen reaction $\bar{\nu}_{e}^{16} O \rightarrow e^{+} N$ with approximately factor 3 smaller cross section than that of $\nu_{e}^{16} O$ [18].

In order to estimate the optimal distance, we compute the expected number of events in neutrino and anti-neutrino channels as well as their ratios as a function of distance with taking into account of neutrino beam energy spread. For definiteness, we assume that the average energy of neutrino beam $\langle E\rangle=100 \mathrm{MeV}$ and beam energy spread of Gaussian type with width $\sigma_{E}=10 \mathrm{MeV}$. We present our results in Fig. 2.

If there is no matter and CP violating phase effects the ratio $R \equiv N\left(\nu_{\mu} \rightarrow \nu_{e}\right) / N\left(\bar{\nu}_{\mu} \rightarrow\right.$ $\left.\bar{\nu}_{e}\right)$ must not vary with the distance but must be a constant which is $\phi_{\nu}\left\langle\sigma_{\nu}\right\rangle / \phi_{\bar{\nu}}\left\langle\sigma_{\bar{\nu}}\right\rangle$ where $\phi_{\nu}\left(\phi_{\bar{\nu}}\right)$ and $\sigma_{\nu}\left(\sigma_{\bar{\nu}}\right)$ are total flux and cross section averaged over neutrino (anti-neutrino) energy, respectively. Taking the error (only statistical one for simplicity) into account, $L$ must not be too short or too long. The optimal distance turned out to be rather short, $L \sim 30-50 \mathrm{~km}$, much shorter than that of the current long-baseline experiments. (see Fig. 2.) At such distance the significance of the $\mathrm{CP}$ violating signal is as large as 3-4 $\sigma$.

Roughly speaking, the deviation of $R$ from the case without $\mathrm{CP}$ violating effect, $\Delta R$, is proportional to $\Delta P$ but what is relevant is the quantity $\Delta P \times N(N$ is the expected number of events in the absence of $\mathrm{CP}$ violating effect) which signals if the $\mathrm{CP}$ violating effect is significant or not. From eq. (3) we can see that when the distance is small so that $\Delta_{13} L \ll 1$, the terms proportinal to $J$ (or $\Delta P$ ) behaves like $\Delta P \propto(L / E)^{3}$. This implies that assuming $\phi_{\nu} \propto 1 / L^{2}, \Delta P \times N \propto \Delta P \sigma(E) / L^{2} \propto \sigma(E) L / E^{3}$ is an increasing function of

${ }^{2}$ Notice that the energy dependence of $\nu_{e}^{16} O$ reaction is very steep below $E=100 \mathrm{MeV}$. For example, it is smaller by an order of magnitude at $E=60 \mathrm{MeV}$. Because the energy dependence of $\Delta P$ is mild we are invited to take larger energy than the resonance energy. 
$L$. When the distance is larger and $\Delta_{13} L$ is not small, $\Delta P \propto(L / E)$ and this implies that $\Delta P \times N \propto \sigma(E) E / L$ is a decreasing function of $L$.

Hence, the significance of $\mathrm{CP}$ violating effect is expected to be maximal at some distance $L$ where $\Delta P \times N$ takes the largest value. In our case it corresponds to $L \sim 30-50 \mathrm{~km}$ for the mixing parameters and neutrino energy we consider, as indicated in Fig. 2.

Let us also make some comments on the validity of our choice of low neutrino energy (as low as $\sim 100 \mathrm{MeV}$ ) from the view point of the expected number of events. Suppose that the relevant cross section $\sigma(E)$ behaves like $\sigma(E) \propto E^{\alpha}$ and we can neglect matter effect. In this case, $N$ is $\sim f(L / E) \times E^{(\alpha-2)}$ where $f(L / E)$ is some function of $L / E$. From this we can conclude that if $\alpha=2$ the same number of events can be expected as long as the value of $L / E$ is the same if the same intensity of neutrino beam is assumed because the increase in the cross section with higher energy can be compensated by the decrese of the flux due to larger distance. On the other hand, if $\alpha \neq 2$ such scaling law does not apply. If $\alpha>2$ $(\alpha<2)$ the one can expect larger number of events with higher (lower) energy if the value of $L / E$ is kept constant. For neutrino energy above $100 \mathrm{MeV}$ but below $1 \mathrm{GeV}$, for neutrno channel, roughly speaking $\alpha \sim 2$ whereas for anti neutrino channel $\alpha \sim 1$ [22]. Therefore, our choice of lower energy seems to be preferable. For energy well above GeV, $\alpha \sim 1$ for both channels and lower energy is preferable.

We conclude that the experiment is quite feasible under such intense neutrino beam and a megaton detector. Fortunately, the possibility of constructing a megaton water Cherenkov detector is already discussed by the experimentalists [21].

Finally, a few remarks are in order:

(1) Imitating vacuum mechanism does work also for the SMA solar MSW solution after averaging over the rapid oscillations due to larger $\Delta m^{2}$. Because of the tiny vacuum angle, however, the number of events is smaller by a factor of $\sim 2500$ than the LMA case and the experiment does not appear feasible with the same apparatus.

(2) An alternative way of measuring $\mathrm{CP}$ violation is the multiple detector method [4] which may be inevitable if either one of $\nu$ or $\bar{\nu}$ beam is difficult to prepare. It utilizes the fact that the first, the second, and the third terms in the oscillation probability (16) have different $L$ dependences, $\sim L$-independent (after averaging over energy spread of the neutrino beam), $\sim L^{2}$, and $\sim L$, respectively, in the linear approximation. []

(3) Intense neutrino beams from muon storage ring at low energies, proposed as PRISM, Phase-Rotation Intense Secondary Mesons [23], would be an ideal source for neutrinos for the experiment proposed in this paper. Of course, it requires identification of $\overline{\nu_{e}}$ from $\nu_{e}$ by some methods, e.g., by adding Chlorine $\left.{ }^{35} \mathrm{Cl}\right)$ into the Water Cherenkov detector to make it sensitive to the characteristic $\sim 8 \mathrm{MeV} \gamma$ rays arising from the absorption of neutron into the Chlorine followed by $\bar{\nu}_{e} p \rightarrow e^{+} n$ reaction.

3 The minimal two detector methods [4] is most powerful if the far (near) detector is placed at $\Delta_{12}=\frac{3 \pi}{2}\left(\frac{\pi}{2}\right)$ because then the difference of the CP violating term in (16) between the far and the near detectors is maximal. However, it requires the baseline length of far detector as $L=6,300\left(\frac{\Delta m_{12}^{2}}{3 \times 10^{-5} \mathrm{eV}^{2}}\right)^{-1}\left(\frac{E}{100 \mathrm{MeV}}\right) \mathrm{km}$ which may or may not be too large to realize. 
(4) We would like to urge experimentalists to think more about the better supermassive detection apparatus than water Cherenkov for highly efficient and accurate measurement of low energy neutrinos.

\section{ACKNOWLEDGMENTS}

We thank Takaaki Kajita, Masayuki Nakahata and Kenzo Nakamura for informative discussions on detection of low energy neutrinos. One of us (HM) thanks Marcelo M. Guzzo and the department of Cosmic Ray and Chronology at Gleb Wataghin Physics Institute in UNICAMP for their hospitality during his visit when the main part of this work was done. This work was supported by the Brazilian funding agency Fundação de Amparo à Pesquisa do Estado de São Paulo (FAPESP), and by the Grant-in-Aid for Scientific Research in Priority Areas No. 11127213, Japanese Ministry of Education, Science, Sports and Culture. 


\section{REFERENCES}

[1] Y. Fukuda et al. (Kamiokande collaboration), Phys. Lett. B335 (1994) 237; Y. Fukuda et al. (SuperKamiokande collaboration), Phys. Rev. Lett. 81 (1998) 1562; T. Kajita, in Neutrino Physics and Astrophysics, Proceedings of the XVIIIth International Conference on Neutrino Physics and Astrophysics (Neutrino '98), June 4-9, 1998, Takayama, Japan, edited by Y. Suzuki and Y. Totsuka, (Elsevier Science B.V., Amsterdam, 1999) page 123.

[2] Homestake Collaboration, K. Lande et al., Astrophys .J. 496, 505 (1998); SAGE Collaboration, J. N. Abdurashitov et al., Phys. Rev. C 60, 055801 (1999); GALLEX Collaboration, W. Hampel et al., Phys. Lett. B 447, 127 (1999); Kamiokande Collaboration, Y. Fukuda et al. Phys. Rev. Lett. 77, 1683 (1996); SuperKamiokande Collaboration, Y. Fukuda et al., Phys. Rev. Lett. 81, 1158 (1998); ibid. 81, 4279 (1998); ibid. 82, 2430 (1999); ibid. 82, 1810 (1999).

[3] M. Kobayashi and T. Maskawa, Prog. Theor. Phys. 49 (1973) 652.

[4] H. Minakata and H. Nunokawa, Phys. Lett. B413 (1997) 369.

[5] H. Minakata and H. Nunokawa, Phys. Rev. D57 (1998) 4403.

[6] N. Cabbibo, Phys. Lett. B72, (1978) 333; V. Barger, K. Wisnant and R. J. N. Phillips, Phys. Rev. Lett. 45 (1980) 2084; S. Pakvasa, in Proceedings of the XXth International Conference on High Energy Physics, edited by L. Durand and L. G. Pondrom, AIP Conf. Proc. No. 68 (AIP, New York, 1981), Vol. 2, pp. 1164.

[7] T. K. Kuo and J. Pantaleone, Phys. Lett. B198 (1987) 406.

[8] J. Arafune and J. Sato, Phys. Rev. D55 (1997) 1653; J. Arafune, M. Koike and J. Sato, Phys. Rev. D56 (1997) 3093; Erratum ibid., D 60 (1999) 119905.

[9] M. Tanimoto, Phys. Rev. D55 (1997) 322; Prog. Theor. Phys. 97 (1997) 901; S. M. Bilenky, C. Giunti and W. Grimus, Phys. Rev. D58 (1998) 033001; A. De Rujula, M. B. Gavela, and P. Hernandez, Nucl. Phys. B547 (1999) 21; K. Dick et al., Nucl. Phys. B562 (1999) 29; A. Gago, V. Pleitez and R. Zukanovich Funchal, Phys. Rev. D61 (2000) 016004 ; A. Donini et al., Nucl. Instrum. Meth. A451 (2000) 58.

[10] S. P. Mikheyev and A. Smirnov, Nuovo Cim. 9C (1986) 17; L. Wolfenstein, Phys. Rev. D17 (1978) 2369.

[11] J.N. Bahcall, P.I. Krastev and A.Yu. Smirnov, Phys. Rev. D60 (1999) 093001; M.C. Gonzalez-Garcia, P.C. de Holanda, C. Peña-Garay and J.W.F. Valle, Nucl. Phys. B573 (2000) 3.

[12] G. L. Fogli, E. Lisi, D. Montanino, and A. Palazzo, Phys. Rev. D62 (2000) 013002.

[13] C. Jarlskog, Phys. Rev. Lett. 55 (1985) 1039.

[14] M. Koike and J. Sato, Phys. Rev. D61 (2000) 073012; hep-ph/9911258.

[15] O. Yasuda, Acta. Phys. Polon. B 30 (1999) 3089 hep-ph/9910428].

[16] T. K. Kuo and J. Pantaleone, Phys. Rev. D35 (1987) 3432; C-S. Lim, in Proceedings of BNL Neutrino Workshop, February 1987, New York, USA, edited by M. J. Murtagh, (BNL-52079, C87/02/05) page 249.

[17] CHOOZ Collaboration, M. Apollonio et al., Phys. Lett. B420 (1998) 397; ibid., B466 (1999) 415.

[18] W. Haxton, Phys. Rev. D36 (1987) 2283.

[19] K. Nishikawa et al., Proposal for a Long Baseline Neutrino Oscillation Experiment using KEK-PS and Super-Kamiokande, 1995; Y. Oyama, hep-ex/9803014. 
[20] Y. Mori, Talk at KEK International Workshop on "High Intensity Muon Sources", KEK, Japan, December 1-4, 1999, to appear in Proceedings.

[21] K. Nakamura, Talk at Workshop on Neutrino Oscillations and Their Origin, Fujiyoshida, Japan, Feb. 11-13, 2000, to appear in Proceedings.

[22] C. H. Llwewllyn Smith, Phys. Rep. 3C (1972), 261; M. Nakahata et al., J. Phys. Soc. Jap., 55 (3786), 1986.

[23] Y. Kuno, in Proceedings of the Workshop on High Intensity Secondary Beam with Phase Rotation, September 21-22, 1998 (Institute for Chemical Research, Kyoto University). 


\section{FIGURES}

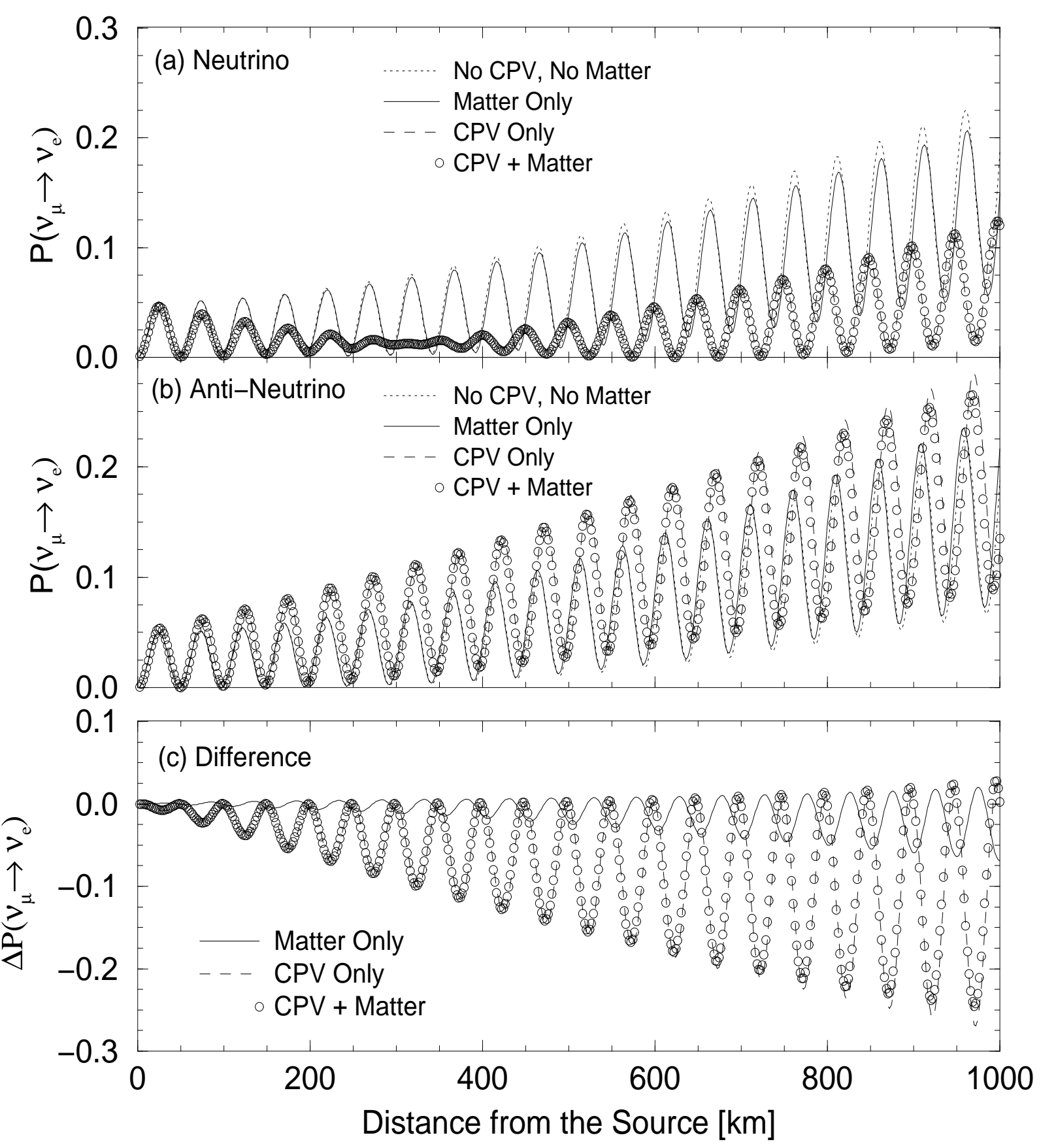

FIG. 1. Oscillation probability for (a) neutrinos, $P\left(\nu_{\mu} \rightarrow \nu_{e}\right)$, (b) anti-neutrinos, $P\left(\bar{\nu}_{\mu} \rightarrow \bar{\nu}_{e}\right)$, and (c) their difference, $\Delta P\left(\nu_{\mu} \rightarrow \nu_{e}\right) \equiv P\left(\nu_{\mu} \rightarrow \nu_{e}\right)-P\left(\bar{\nu}_{\mu} \rightarrow \bar{\nu}_{e}\right)$ with fixed neutrino energy $E_{\nu}=$ $60 \mathrm{MeV}$, are plotted as a function of distance from the source. The mixing parameters are fixed to be $\Delta m_{13}^{2}=3 \times 10^{-3} \mathrm{eV}^{2}, \sin ^{2} 2 \theta_{23}=1.0, \Delta m_{12}^{2}=2.7 \times 10^{-5} \mathrm{eV}^{2}, \sin ^{2} 2 \theta_{12}=0.79, \sin ^{2} 2 \theta_{13}=0.1$ and $\delta=\pi / 2$. We take the matter density as $\rho=2.72 \mathrm{~g} / \mathrm{cm}^{3}$ and the electron fraction as $Y_{e}=0.5$. 

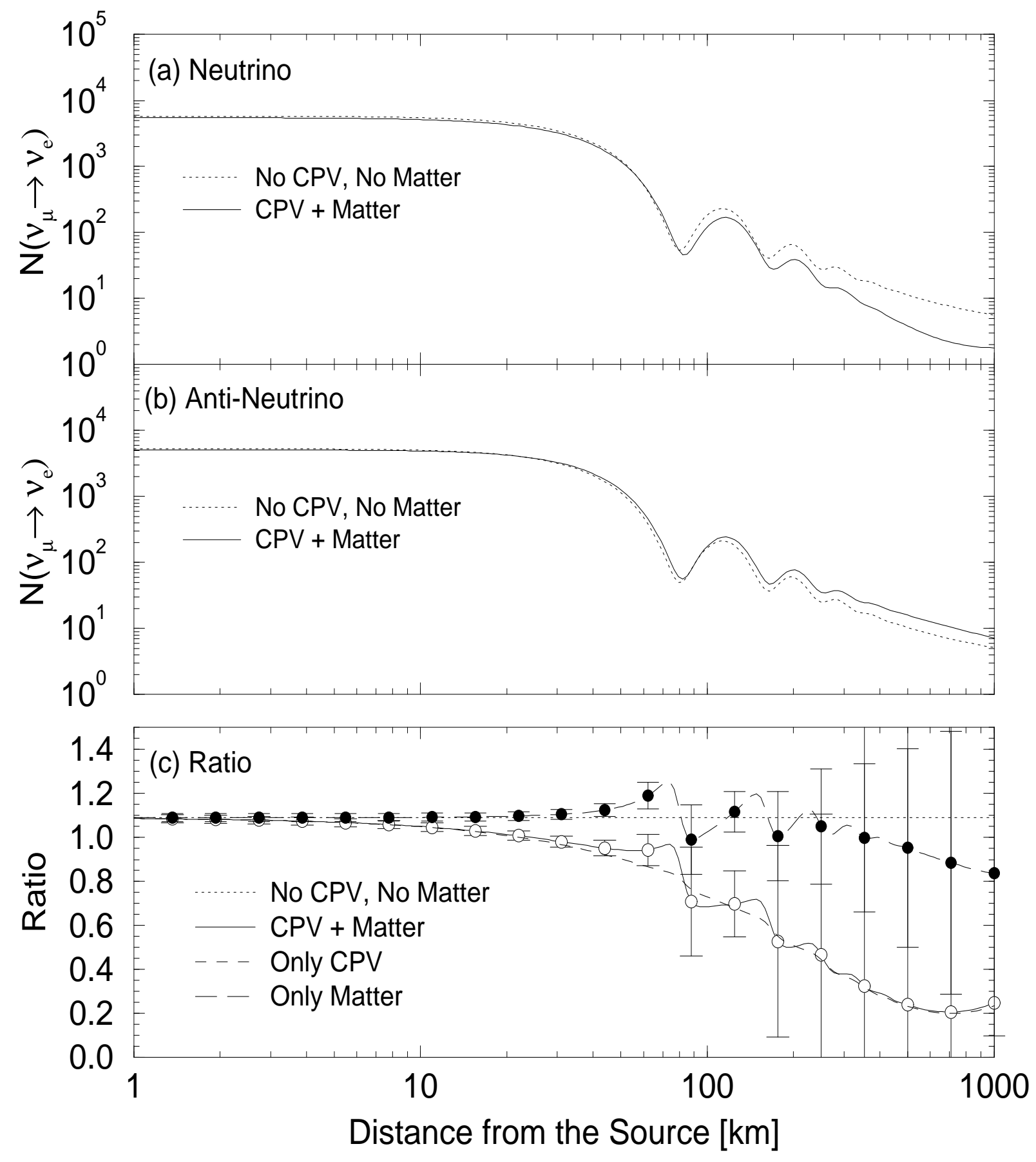

FIG. 2. Expected number of events for (a) neutrinos, $N\left(\nu_{\mu} \rightarrow \nu_{e}\right)$, (b) anti-neutrinos, $N\left(\bar{\nu}_{\mu} \rightarrow \bar{\nu}_{e}\right)$, and (c) their ratio $R \equiv N\left(\nu_{\mu} \rightarrow \nu_{e}\right) / N\left(\bar{\nu}_{\mu} \rightarrow \bar{\nu}_{e}\right)$ with a Gaussian type neutrino energy beam with $\left\langle E_{\nu}\right\rangle=100 \mathrm{MeV}$ with $\sigma=10 \mathrm{MeV}$ are plotted as a function of distance from the source. Neutrion fluxes are assumed to vary as $\sim 1 / L^{2}$ in all the distance range we consider. The mixing parameters as well as the electron number density are fixed to be the same as in Fig. 1. The error bars are only statistical. 